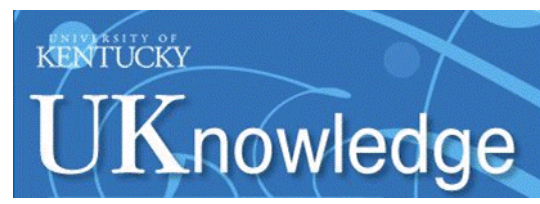

University of Kentucky

UKnowledge

\title{
Timing and Magnitude of Lumbar Spine Contribution to Trunk Forward Bending and Backward Return in Patients with Acute Low Back Pain
}

\author{
Iman Shojaei \\ University of Kentucky, shojaei.iman@gmail.com \\ Milad Vazirian \\ University of Kentucky, milad.vazirian@uky.edu \\ Elizabeth G. Salt \\ University of Kentucky, elizabeth.salt@uky.edu \\ Linda R. Van Dillen \\ Washington University in St. Louis \\ Babak Bazrgari \\ University of Kentucky, babak.bazrgari@uky.edu
}

Follow this and additional works at: https://uknowledge.uky.edu/cbme_facpub

Part of the Biomedical Engineering and Bioengineering Commons

Right click to open a feedback form in a new tab to let us know how this document benefits you.

\section{Repository Citation}

Shojaei, Iman; Vazirian, Milad; Salt, Elizabeth G.; Van Dillen, Linda R.; and Bazrgari, Babak, "Timing and Magnitude of Lumbar Spine Contribution to Trunk Forward Bending and Backward Return in Patients with Acute Low Back Pain" (2017). Biomedical Engineering Faculty Publications. 35.

https://uknowledge.uky.edu/cbme_facpub/35

This Article is brought to you for free and open access by the Biomedical Engineering at UKnowledge. It has been accepted for inclusion in Biomedical Engineering Faculty Publications by an authorized administrator of UKnowledge. For more information, please contact UKnowledge@lsv.uky.edu. 


\section{Timing and Magnitude of Lumbar Spine Contribution to Trunk Forward Bending and Backward Return in Patients with Acute Low Back Pain}

\section{Digital Object Identifier (DOI)}

https://doi.org/10.1016/j.jbiomech.2016.12.039

\section{Notes/Citation Information}

Published in Journal of Biomechanics, v. 53, p. 71-77.

(C) 2017 Elsevier Ltd. All rights reserved.

This manuscript version is made available under the CC-BY-NC-ND 4.0 license https://creativecommons.org/licenses/by-nc-nd/4.0/.

The document available for download is the author's post-peer-review final draft of the article. 


\title{
Timing and Magnitude of Lumbar Spine Contribution to Trunk Forward Bending and Backward Return in Patients with Acute Low Back Pain
}

\author{
Iman Shojaei ${ }^{1}$, Milad Vazirian ${ }^{1}$, Elizabeth G Salt ${ }^{2}$, Linda R Van Dillen ${ }^{3}$, and Babak \\ Bazrgari ${ }^{1, *}$ \\ ${ }^{1}$ F. Joseph Halcomb III, M.D. Department of Biomedical Engineering, University of Kentucky, \\ Lexington, KY 40506, USA \\ ${ }^{2}$ College of Nursing, University of Kentucky, Lexington, KY 40506, USA \\ ${ }^{3}$ Program in Physical Therapy, Department of Orthopedic Surgery, Washington University School \\ of Medicine, St. Louis, MO 63108, USA
}

\begin{abstract}
Alterations in the lumbo-pelvic coordination denote changes in neuromuscular control of trunk motion as well as load sharing between passive and active tissues in the lower back. Differences in timing and magnitude aspects of lumbo-pelvic coordination between patients with chronic low back pain (LBP) and asymptomatic individuals have been reported; yet, the literature on lumbopelvic coordination in patients with acute LBP is scant. A case-control study was conducted to explore the differences in timing and magnitude aspects of lumbo-pelvic coordination between females with $(n=19)$ and without $(n=19)$ acute LBP. Participants in each group completed one experimental session wherein they performed trunk forward bending and backward return at preferred and fast paces. The amount of lumbar contribution to trunk motion (as the magnitude aspect) as well as the mean absolute relative phase (MARP) and deviation phase (DP) between thoracic and pelvic rotations (as the timing aspect) of lumbo-pelvic coordination were calculated. The lumbar contribution to trunk motion in the $2^{\text {nd }}$ and the $3^{\text {rd }}$ quarters of both forward bending and backward return phases was significantly smaller in the patient than the control group. The MARP and the DP were smaller in the patient vs. the control group during entire motion. The reduced lumbar contribution to trunk motion as well as the more in-phase and less variable lumbopelvic coordination in patients with acute LBP compared to the asymptomatic controls is likely the result of a neuromuscular adaptation to reduce painful deformation and to protect injured lower back tissues.
\end{abstract}

\footnotetext{
*Correspondence to: F. Joseph Halcomb III, M.D. Department of Biomedical Engineering, University of Kentucky, 514E Robotic and Manufacturing Building, Lexington, KY 40506. Tel.: +859 257 1379; fax: +859 257 1856. babak.bazrgari@uky.edu.

CONFLICT OF INTEREST STATEMENT

We declare that all authors have no financial or personal relationships with other persons or organizations that might inappropriately influence our work presented therein.

Publisher's Disclaimer: This is a PDF file of an unedited manuscript that has been accepted for publication. As a service to our customers we are providing this early version of the manuscript. The manuscript will undergo copyediting, typesetting, and review of the resulting proof before it is published in its final citable form. Please note that during the production process errors may be discovered which could affect the content, and all legal disclaimers that apply to the journal pertain.
} 


\section{Keywords}

Acute low back pain; Trunk forward bending and backward return; Lumbo-pelvic coordination; Timing and magnitude aspects; Continuous relative phase

\section{INTRODUCTION}

Lumbo-pelvic coordination during trunk forward bending and backward return is often assessed by clinicians to better identify biomechanical abnormalities in patients with low back pain (LBP) (Esola et al., 1996; Hestœk and Leboeuf-Yde, 2000; Whittaker, 2007). Alterations in lumbo-pelvic coordination denote changes in neuromuscular control of trunk motion as well as changes in the load sharing between passive and active components of the lower back (Davis and Jorgensen, 2005; Davis et al., 1965; Farfan, 1975; Hashemirad et al., 2010). Both neuromuscular control and load sharing have been recognized to play a role in LBP development (Abouhossein et al., 2011; Dubois et al., 2011; Hashemirad et al., 2009; Leinonen, 2003; Panjabi, 2003; van Dieën and Nussbaum, 2000). The assessment of lumbopelvic coordination may simply involve evaluation of the relative contributions of lumbar flexion and pelvic rotation to trunk motion at the end range of forward bending or may include more in-depth evaluation of timing and magnitude of such relative contributions throughout the course of motion (Kim et al., 2013; Lariviere et al., 2000; Mokhtarinia et al., 2016; Pries et al., 2015; Silfies et al., 2009).

In asymptomatic individuals, the lumbar contribution to forward bending has been reported to be dominant in the early stage of trunk motion, whereas pelvis contribution increases toward the end of motion and is dominant at the late stage of motion (Esola et al., 1996; Lee and Wong, 2002; Pal et al., 2007; Tafazzol et al., 2014; Vazirian et al., 2016a; Vazirian et al., 2017; Vazirian et al., 2016b). Conversely, backward return starts with a small lumbar contribution that gradually increases toward the end of motion (Granata and Sanford, 2000; Lee and Wong, 2002; McClure et al., 1997; Pal et al., 2007). In terms of timing of motion, it has been reported that in forward bending, lumbar motion tends to start sooner than pelvic motion and lumbar motion remains ahead of pelvic motion throughout the forward bending. In the backward return lumbar motion remains behind pelvic motion (Pal et al., 2007; Thomas and Gibson, 2007). Compared to asymptomatic individuals, lumbo-pelvic coordination in patients with LBP is generally more in-phase and less variable and involves smaller lumbar contribution to the trunk motion (Mokhtarinia et al., 2016; Seay et al., 2011; Selles et al., 2001). There are, however, some exceptions to such general trend of observed differences in the literature which could be due to heterogeneity of LBP (e.g., different subtypes of LBP), differences in patient's personal characteristics, and difference in performing forward bending and backward return (e.g., pace of task, presence of load, etc.) (Granata and Sanford, 2000; Kim et al., 2013; Silfies et al., 2009; Van Wingerden et al., 2008; Vazirian et al., 2016b). Kim et al. (2013), for instance, observed larger lumbar contribution to the trunk motion in a subgroup of patient with LBP who were identified to have "lumbar flexion with rotation syndrome". Silfies et al. (2009) reported a less in-phase and more variable lumbo-pelvic coordination in patients with LBP compared to asymptomatic controls under a reaching task. Despite considerable research related to 
lumbo-pelvic coordination, most of prior studies included patients with chronic LBP and it is not clear whether their findings can be generalized to include also patients with acute LBP. Due to the simplicity of the assessment, an evaluation of lumbo-pelvic coordination in clinical practice could prove useful to identify biomechanical etiologies for LBP and to direct patient treatment; thus a further understanding of this construct in acute LBP is needed.

Authors of the present study have recently reported the differences in lumbo-pelvic coordination between patients with acute LBP and asymptomatic controls by calculation of the relative contributions of lumbar and pelvis to trunk motion at the end point of forward bending. Compared to asymptomatic controls, patients with LBP implemented smaller lumbar flexion and larger pelvic rotation when bending from standing posture to the end point of forward bending. These results clearly distinguished patients from asymptomatic controls in discrete end points, however, they don't offer any information related to potential differences in lumbo-pelvic coordination throughout the trunk forward bending and backward motion. Further characterization of lumbo-pelvic coordination throughout the entire task cycle could provide more in-depth information about the impact of acute LBP on timing and magnitude aspects of lumbo-pelvic coordination (Mokhtarinia et al., 2016; Pal et al., 2007; Selles et al., 2001; Thomas and Gibson, 2007; Vazirian et al., 2016b). In other words, potential biomechanical abnormalities in the lower back of patients with LBP, particularly due to neuromuscular impairments, could be better identified by assessment of lumbo-pelvic coordination throughout the entire task. Therefore, the objective of this study was to investigate differences in timing and magnitude aspects of lumbo-pelvic coordination between patients with acute LBP and asymptomatic controls during forward bending and backward return. Lumbar contribution to the trunk rotation was investigated at each quartile of forward bending and backward return as the magnitude aspect of lumbo-pelvic coordination. The timing aspect of lumbo-pelvic coordination was investigated using the continuous relative phase method (Lamb and Stöckl, 2014). We hypothesized that patients with acute LBP would display a more in-phase and less variable lumbo-pelvic coordination that involves a reduced lumbar contribution to the trunk motion compared to the asymptomatic controls during the entire period of the forward bending and backward return task.

\section{METHODS}

\section{Study Design and Participants}

A case-control study design was used wherein 19 female patients (aged 40-70 years old) with acute LBP (health care provider-diagnosed LBP $\leq 3$ months) completed a set of trunk forward bending and backward return tasks. Data for 19 asymptomatic female controls (aged 40-70 years old) were extracted from an earlier study (Vazirian et al., 2016a). All participants completed an informed consent procedure approved by the University of Kentucky Institutional Review Board before participation. Age, stature, body mass, and body mass index (BMI) for the two groups were comparable (Table.1). Asymptomatic controls with any history of LBP or musculoskeletal disorders were excluded (Shojaei et al., 
2016; Vazirian et al., 2016a). Patients with acute LBP were excluded if they had any significant cognitive impairment, intention to harm themselves or others, or substance abuse.

\section{Experimental Procedures}

Two wireless Inertial Measurement Units (IMUs; Xsens Technologies, Enschede, Netherlands) were attached superficial to the $\mathrm{T} 10$ and the $\mathrm{S} 1$ spinous process of participants to collect kinematics of thorax and pelvis as rigid bodies $(50 \mathrm{~Hz})$. A Kalman filter was used to minimize any potential effect of noise on the data. Each participant completed two trunk forward bending and backward return tasks in the sagittal plane; one at a preferred pace and the other at a fast pace. During the task with preferred pace, participants stood in an upright posture for 5 seconds, bent forward using a preferred pace to reach their maximum trunk rotation, held their maximum trunk rotation for 5 seconds, returned back to the initial upright position, and stood again for 5 seconds. During the task with fast pace, participants performed the same task but with their fastest possible pace and without a pause at the maximum trunk rotation. Each task pace was repeated three times, and participants completed the task with the preferred pace prior to the task with the fast pace.

\section{Data analysis}

Using the kinematics data collected with the IMUs, pelvic and thoracic rotations were found with respect to the standing posture. At each time instant, flexion/extension of lumbar spine (i.e., as a deformable segment between thorax and pelvis) was calculated as the difference between the pelvic and thoracic rotations. To calculate the lumbar contribution, the forward bending and the backward return of each task was divided into quarters of equal thoracic rotation. The ratio of range of lumbar flexion/extension over the range of thoracic rotation was then calculated for each quartile. Lumbar contribution in each quartile of forward bending and backward return task was finally calculated as the average of the above ratio for the same quartile across the three repetitions of the task. The thoracic and pelvic rotation data were also used to calculate the continuous relative phase between thorax and pelvis by initially generating the phase planes of pelvic and thoracic rotations according to (Lamb and Stöckl, 2014), and then subtracting the pelvic phase angle from the thoracic phase angle at each instant of the task. To characterize the timing aspect of lumbo-pelvic coordination, two measures from the continuous relative phase (CRP) curve of forward bending and backward return were extracted: 1) the mean absolute relative phase (MARP), and 2) the deviation phase (DP) (Stergiou et al., 2001). Briefly, the mean and standard deviation of the absolute value of relative phase for each percentile of trunk forward bending and backward return across the three repetitions of each task were initially obtained. Subsequently, the average of the calculated mean and standard deviation values over the entire forward bending and backward return were respectively calculated as MARP and DP values. By definition, MARP values closer to 0 represent a more "in-phase" lumbo-pelvic coordination (i.e., more synchronous movement of segments) whereas values closer to $\pi$ radians represent a more "out-of-phase" lumbo-pelvic coordination (i.e., less synchronous movement of segments). Moreover, a smaller DP represents a lumbo-pelvic coordination with less trial-to-trial variability (i.e., a more stable motion pattern). 


\section{Statistical Analysis}

For each task and phase of trunk motion (i.e., forward bending and backward return), the lumbar contribution in each quartile, MARP, and DP were extracted for statistical analyses. All statistical procedures were conducted in SPSS (IBM SPSS Statistics 23, Armonk, NY, USA), and in all cases a $p$ value smaller than 0.05 was considered as statistically significant. Mixed-model analysis of variance (ANOVA) tests were conducted on the dependent variables with group (with and without LBP) and age (40-50, 50-60, 60-70) as the betweensubjects factors and motion pace (preferred and fast) as the within-subjects factor. Mixedmodel ANOVA assumptions were verified, and significant ANOVA tests were followed by post hoc tests using Tukey's procedure.

\section{RESULTS}

\section{Interaction effects}

Forward bending-The lumbar contribution in the $1^{\text {st }}$ quarter was larger (40-50: $F=4.95$, $p=0.045 ; 60-70: F=7.90, p=0.016)$ in the control vs. patient group only during the task with fast pace and for individuals in the 40-50 (40s) and 60-70 (60s) year-old age groups (Fig. 1). This lumbar contribution was also larger $(F=10.47, p=0.018)$ in the task with preferred vs. fast pace only for patients in the 60-70 (60s) year-old age group (Fig. 1).

Additionally, lumbar contribution in the $4^{\text {th }}$ quarter was larger $(F=6.22, p=0.041)$ in the task with preferred vs. fast pace only for patients in the 50-60 (50s) year-old age group. This lumbar contribution was also larger $(F=5.97, p=0.012)$ in the 60-70 (60s) versus 50-60 (50s) year-old age group only among patients and under task with fast pace (Fig. 2).

Backward return-The lumbar contribution in the $1^{\text {st }}$ quarter was larger $(F=14.71$, $p=0.012 ; F=9.37, p=0.022)$ during the task with preferred vs. fast pace only for controls in the 40-50 (40s) and 60-70 (60s) year-old age group (Fig. 3). This lumbar contribution was also larger $(F=5.01, p=0.020)$ for controls in the $40-50$ (40s) vs. controls in the 50-60 (50s) year-old age group (Fig. 3).

Additionally, the lumbar contribution in the $4^{\text {th }}$ quarter was larger $(F=5.12, p=0.043)$ for controls in the 60-70 (60s) year-old age group vs. patients in the same age group only during the task with fast pace (Fig. 4). This lumbar contribution was also larger $(F=17.62$, $p=0.009$ ) in the task with preferred vs. fast pace only for controls in the 50-60 (50s) year-old age group (Fig. 4). Furthermore, this lumbar contribution was larger $(F=21.26, p=0.004)$ during the task with preferred vs. fast pace only for patients in the 60-70 (60s) year-old age group (Fig. 4).

Group differences-During forward bending and backward return, the lumbar contribution in the $2^{\text {nd }}$ and $3^{\text {rd }}$ quarters was smaller in the patient group than the control group (Table 2 and Table 3). Furthermore, the MARP and DP were smaller in the patient vs. control group during forward bending and backward return (Table 2 and Table 3 ).

The effects of task pace-Lumbar contribution to the trunk rotation was smaller during the $2^{\text {nd }}$ and $3^{\text {rd }}$ quarters of both forward bending and backward return of the task with fast 
vs. preferred pace (Table 3). MARP during forward bending and DP during both forward bending and backward return were smaller in the task with fast vs. preferred pace (Table 2 and Table 3).

Age-related differences-Lumbar contribution to the trunk rotation during the $2^{\text {nd }}$ and $3^{\text {rd }}$ quarters of both forward bending and backward return was larger in the 40-50 (40s) year-old age group than the other two age groups (Table 3). No age related differences in MARP and DP during forward bending and backward return were found (Table 2 and Table $3)$.

\section{DISCUSSION}

The purpose of this study was to investigate differences in magnitude and timing aspects of lumbo-pelvic coordination between patients with acute LBP and asymptomatic controls during trunk forward bending and backward return. Lumbar contribution to the trunk motion in the $2^{\text {nd }}$ and $3^{\text {rd }}$ quarters of forward bending and backward return were smaller in patients with acute LBP vs. asymptomatic controls (i.e., partially confirming our hypothesis). Lumbo-pelvic coordination was more in-phase (i.e., denoted by smaller MARP values) and less variable (i.e., denoted by smaller DP values) in patients with acute LBP vs. asymptomatic controls (i.e., confirming our hypothesis).

In our earlier study, lumbar contribution to the trunk motion at the end point of the forward bending was observed to be smaller in patients with acute LBP vs. asymptomatic controls. Our current finding, further suggest that such overall observed difference was due to smaller lumbar contribution in patients with acute LBP in the $2^{\text {nd }}$ and $3^{\text {rd }}$ quarters of forward bending and backward return. To the best of our knowledge, no other study has reported differences in lumbar contribution to trunk motion throughout the forward bending and backward return between patients with acute LBP and asymptomatic controls. However, in studies including patients with chronic LBP and individuals with a history of LBP similarly smaller lumbar contribution to the trunk motion in all quartiles (Lariviere et al., 2000) as well as in the early stage (Porter and Wilkinson, 1997) or middle stage (Esola et al., 1996) of forward bending and backward return have been reported.

While in the current study the patients implemented a more in-phase motion of pelvis and thorax segments compared to asymptomatic controls, Wong and Lee (2004) reported no differences in timing aspects of the lumbo-pelvic coordination between a patient group and a asymptomatic control group. Such inconsistency in the results between the current study and the study by Wong and Lee (2004) could be due to the differences in the personal characteristics of participants (e.g., females $\sim 57$ years old in the current study vs. males 40 years old in the study by Wong and Lee (2004)), different methods of data analysis (CRP method in the current study vs. Cross-correlation method in the study by Wong and Lee (2004); see Vazirian et al. (2016b) for differences between the two methods) and potential differences in LBP subtypes. In studies including patients with chronic LBP, there are reports of a more in-phase lumbo-pelvic coordination in patients vs. asymptomatic controls during forward bending and backward return (Asgari et al., 2015; Mokhtarinia et al., 2016) as well as during walking and running (Seay et al., 2011; Selles et al., 2001). In contrast, 
Silfies et al. (2009) and Paquet et al. (1994) reported, respectively, a more out-of-phase and similar lumbo-pelvic coordination in patients vs. asymptomatic controls. Discrepancies in the results of studies concerning patients with chronic LBP may be attributed to the differences in the population studied (e.g., personal characteristics and LBP subtype), differences in the methods of data analysis, and differences in LBP severity at the time of study.

The smaller contribution of lumbar flexion to trunk motion, as seen in patients with acute LBP in the current study, reduces passive contribution of lower back tissues in offsetting the physical demand of the task on the lower back. Such an alteration in lumbar contribution has been suggested to prevent painful deformation in posterior elements of the ligamentous spine (Colloca and Hinrichs, 2005). More in-phase and less variable lumbo-pelvic coordination, also known as phase-locked or rigid coordination (Mokhtarinia et al., 2016), is regarded as a protective motor control strategy to reduce the likelihood of painful deformation of spinal tissues under dynamic tasks. Such a strategy, however, demands higher levels of trunk muscles activation and co-activation which in turn can lead to increased spinal loads and muscle fatigue (Bazrgari et al., 2008a, b; Marras et al., 2001).

The lumbar contribution in the $2^{\text {nd }}$ and $3^{\text {rd }}$ quarters was smaller during the task with fast vs. preferred pace for both forward bending and backward return. Similarly, MARP and DP were found to be smaller during the task with fast pace. These findings are consistent with earlier reports on the effects of task pace on trunk kinematics variability (Asgari et al., 2015). The smaller lumbar contribution and more in-phase lumbo-pelvic coordination is consistent with the strategy to prevent painful deformation and injury (intensified by viscoelastic behavior and inertial demand of fast tasks) given the higher risk of injury under fast trunk motion (Bazrgari et al., 2008c).

Better understanding of differences in lumbo-pelvic coordination during trunk forward bending and backward return between individuals with and without LBP is clinically important (Panjabi, 2003; Van Hoof et al., 2012; White III and Panjabi, 1978). Specifically, quantification of such differences, as done in the present study, may improve the effectiveness of current management paradigm for LBP by positively impacting the diagnosis and treatment stages. More in-depth information about normal and abnormal trunk kinematics during trunk forward bending and backward return can help better match patient pathology with targeted treatments and decide whether a given treatment is moving the patient in the right direction. Additionally, our results indicate that lumbo-pelvic coordination varies with age which also should be considered in the diagnostic process.

Although our findings contribute to the current understanding of the timing and magnitude of lumbar spine contribution to the trunk forward bending and backward return in patients with acute LBP, there are study limitations. First due the use of cross sectional data, we are unable to infer causality. As such we are unable to infer if study findings result in or are consequence to acute LBP. Second, we did not control for intersubject variability such as anthropometric measures, pain level, potential musculoskeletal abnormalities like foot shape abnormalities, flat back, hyper-lordosis as well as LBP-related disability, fear of movement, and general health status. With this being said, there is the possibility of additional unknown 
factors that affect study outcomes and were not included in our analysis. Third, although we controlled for age and gender-related differences, the influence of differences in lumbar spine stiffness or mobility between groups on our finding, though perhaps minimal, should not be overlooked. Finally, while studying magnitude and timing aspects of lumbo-pelvic coordination in patients with acute LBP provides some insights into neuromuscular control of trunk motion and load sharing between lower back tissues, quantification of such variables requires detailed model-based studies (Arjmand et al., 2009; Bazrgari et al., 2008a) which we plan to conduct in the future.

In summary, the lumbar contribution to trunk motion during the $2^{\text {nd }}$ and $3^{\text {rd }}$ quarters of trunk forward bending and backward return phases of motion as well as MARP and the DP during the entire motion were smaller in the patient vs. the control group. These differences in lumbo-pelvic coordination of individuals with acute versus without acute LBP are likely to be due to a neuromuscular motor control strategy to temporarily reduce the painful deformations in the lumbar tissues.

\section{Acknowledgments}

This work was supported in part by the National Center for Research Resources and the National Center for Advancing Translational Sciences [UL1TR000117]. Dr. Van Dillen's contribution was supported by grant NICHD/ NCMRR R01 HD047709. The content of this manuscript is solely the responsibility of the authors and does not necessarily represent the official views of the NIH.

\section{References}

Abouhossein A, Weisse B, Ferguson SJ. A multibody modelling approach to determine load sharing between passive elements of the lumbar spine. Comput Methods Biomech Biomed Engin. 2011; 14:527-537. [PubMed: 21128134]

Arjmand N, Gagnon D, Plamondon A, Shirazi-Adl A, Lariviere C. Comparison of trunk muscle forces and spinal loads estimated by two biomechanical models. Clin Biomech. 2009; 24:533-541.

Asgari M, Sanjari MA, Mokhtarinia HR, Sedeh SM, Khalaf K, Parnianpour M. The effects of movement speed on kinematic variability and dynamic stability of the trunk in healthy individuals and low back pain patients. Clin Biomech. 2015; 30:682-688.

Bazrgari B, Shirazi-Adl A, Kasra M. Computation of trunk muscle forces, spinal loads and stability in whole-body vibration. J Sound Vib. 2008a; 318:1334-1347.

Bazrgari B, Shirazi-Adl A, Kasra M. Seated whole body vibrations with high-magnitude accelerations -relative roles of inertia and muscle forces. J Biomech. 2008b; 41:2639-2646. [PubMed: 18672242]

Bazrgari B, Shirazi-Adl A, Trottier M, Mathieu P. Computation of trunk equilibrium and stability in free flexion-extension movements at different velocities. J Biomech. 2008c; 41:412-421. [PubMed: 17897654]

Colloca CJ, Hinrichs RN. The biomechanical and clinical significance of the lumbar erector spinae flexion-relaxation phenomenon: a review of literature. J Manipulative Physiol Ther. 2005; 28:623631. [PubMed: 16226632]

Davis KG, Jorgensen MJ. Biomechanical modeling for understanding of low back injuries: a systematic review. Occupational Ergonomics. 2005; 5:57-76.

Davis PR, Troup J, Burnard J. Movements of the thoracic and lumbar spine when lifting: a chronocyclophotographic study. J Anat. 1965; 99:13. [PubMed: 14245340]

Dubois JD, Piché M, Cantin V, Descarreaux M. Effect of experimental low back pain on neuromuscular control of the trunk in healthy volunteers and patients with chronic low back pain. $J$ Electromyogr Kinesiol. 2011; 21:774-781. [PubMed: 21641235] 
Esola MA, McClure PW, Fitzgerald GK, Siegler S. Analysis of lumbar spine and hip motion during forward bending in subjects with and without a history of low back pain. Spine (Phila Pa 1976). 1996; 21:71-78. [PubMed: 9122766]

Farfan H. Muscular mechanism of the lumbar spine and the position of power and efficiency. Orthop Clin North Am. 1975; 6:135-144. [PubMed: 123048]

Granata KP, Sanford AH. Lumbar-pelvic coordination is influenced by lifting task parameters. Spine (Phila Pa 1976). 2000; 25:1413-1418. [PubMed: 10828924]

Hashemirad F, Talebian S, Hatef B, Kahlaee AH. The relationship between flexibility and EMG activity pattern of the erector spinae muscles during trunk flexion-extension. J Electromyogr Kinesiol. 2009; 19:746-753. [PubMed: 18400517]

Hashemirad F, Talebian S, Olyaei GR, Hatef B. Compensatory behavior of the postural control system to flexion-relaxation phenomena. J Bodyw Mov Ther. 2010; 14:418-423. [PubMed: 20850051]

Hestœk L, Leboeuf-Yde C. Are chiropractic tests for the lumbo-pelvic spine reliable and valid? A systematic critical literature review. J Manipulative Physiol Ther. 2000; 23:258-275. [PubMed: 10820299]

Kim, M-h, Yi, C-h, Kwon, O-y, Cho, S-h, Cynn, H-s, Kim, Y-h, Hwang, S-h, Choi, B-r, Hong, J-a, Jung, D-h. Comparison of lumbopelvic rhythm and flexion-relaxation response between 2 different low back pain subtypes. Spine (Phila Pa 1976). 2013; 38:1260-1267. [PubMed: 23514875]

Lamb PF, Stöckl M. On the use of continuous relative phase: Review of current approaches and outline for a new standard. Clin Biomech. 2014; 29:484-493.

Lariviere C, Gagnon D, Loisel P. The effect of load on the coordination of the trunk for subjects with and without chronic low back pain during flexion-extension and lateral bending tasks. Clin Biomech. 2000; 15:407-416.

Lee RY, Wong TK. Relationship between the movements of the lumbar spine and hip. Hum Mov Sci. 2002; 21:481-494. [PubMed: 12450680]

Leinonen, V. Kuopion Yliopisto. 2003. Neuromuscular control in lumbar disorders.

Marras WS, Davis KG, Ferguson SA, Lucas BR, Gupta P. Spine loading characteristics of patients with low back pain compared with asymptomatic individuals. Spine (Phila Pa 1976). 2001; 26:2566-2574. [PubMed: 11725237]

McClure PW, Esola M, Schreier R, Siegler S. Kinematic analysis of lumbar and hip motion while rising from a forward, flexed position in patients with and without a history of low back pain. Spine (Phila Pa 1976). 1997; 22:552-558. [PubMed: 9076888]

Mokhtarinia HR, Sanjari MA, Chehrehrazi M, Kahrizi S, Parnianpour M. Trunk coordination in healthy and chronic nonspecific low back pain subjects during repetitive flexion-extension tasks: Effects of movement asymmetry, velocity and load. Hum Mov Sci. 2016; 45:182-192. [PubMed: 26684726]

Pal P, Milosavljevic S, Sole G, Johnson G. Hip and lumbar continuous motion characteristics during flexion and return in young healthy males. Eur Spine J. 2007; 16:741-747. [PubMed: 16924552]

Panjabi MM. Clinical spinal instability and low back pain. J Electromyogr Kinesiol. 2003; 13:371379. [PubMed: 12832167]

Porter JL, Wilkinson A. Lumbar-hip flexion motion: a comparative study between asymptomatic and chronic low back pain in 18-to 36-year-old men. Spine (Phila Pa 1976). 1997; 22:1508-1513. [PubMed: 9231971]

Pries E, Dreischarf M, Bashkuev M, Putzier M, Schmidt H. The effects of age and gender on the lumbopelvic rhythm in the sagittal plane in 309 subjects. J Biomech. 2015; 48:3080-3087. [PubMed: 26294355]

Seay JF, Van Emmerik RE, Hamill J. Low back pain status affects pelvis-trunk coordination and variability during walking and running. Clin Biomech. 2011; 26:572-578.

Selles RW, Wagenaar RC, Smit TH, Wuisman PI. Disorders in trunk rotation during walking in patients with low back pain: a dynamical systems approach. Clin Biomech. 2001; 16:175-181.

Shojaei I, Vazirian M, Croft E, Nussbaum MA, Bazrgari B. Age related differences in mechanical demands imposed on the lower back by manual material handling tasks. J Biomech. 2016; 49:896903. [PubMed: 26556714] 
Silfies SP, Bhattacharya A, Biely S, Smith SS, Giszter S. Trunk control during standing reach: A dynamical system analysis of movement strategies in patients with mechanical low back pain. Gait Posture. 2009; 29:370-376. [PubMed: 19046882]

Stergiou N, Jensen JL, Bates BT, Scholten SD, Tzetzis G. A dynamical systems investigation of lower extremity coordination during running over obstacles. Clin Biomech. 2001; 16:213-221.

Tafazzol A, Arjmand N, Shirazi-Adl A, Parnianpour M. Lumbopelvic rhythm during forward and backward sagittal trunk rotations: combined in vivo measurement with inertial tracking device and biomechanical modeling. Clin Biomech. 2014; 29:7-13.

Thomas JS, Gibson GE. Coordination and timing of spine and hip joints during full body reaching tasks. Hum Mov Sci. 2007; 26:124-140. [PubMed: 17055098]

Van Dieën, JH., Nussbaum, MA. Year Occupational biomechanics of the low back. Proceedings of the Human Factors and Ergonomics Society... Annual Meeting;

Van Wingerden JP, Vleeming A, Ronchetti I. Differences in standing and forward bending in women with chronic low back or pelvic girdle pain: indications for physical compensation strategies. Spine (Phila Pa 1976). 2008; 33:E334-E341. [PubMed: 18469680]

Vazirian M, Shojaei I, Agarwal A, Bazrgari B. Lumbar Contribution to the Trunk Forward Bending and Backward Return; Age-related Differences. Ergonomics. 2016a:1-26.

Vazirian M, Shojaei I, Bazrgari B. Age-related differences in the timing aspect of lumbopelvic rhythm during trunk motion in the sagittal plane. Hum Mov Sci. 2017; 51:1-8. [PubMed: 27814563]

Vazirian M, Van Dillen L, Bazrgari B. Lumbopelvic rhythm during trunk motion in the sagittal plane: A review of the kinematic measurement methods and characterization approaches. Physical Therapy and Rehabilitation. 2016b; 3:5.

Whittaker, JL. Ultrasound imaging for rehabilitation of the lumbopelvic region: a clinical approach. Elsevier Health Sciences; 2007. 

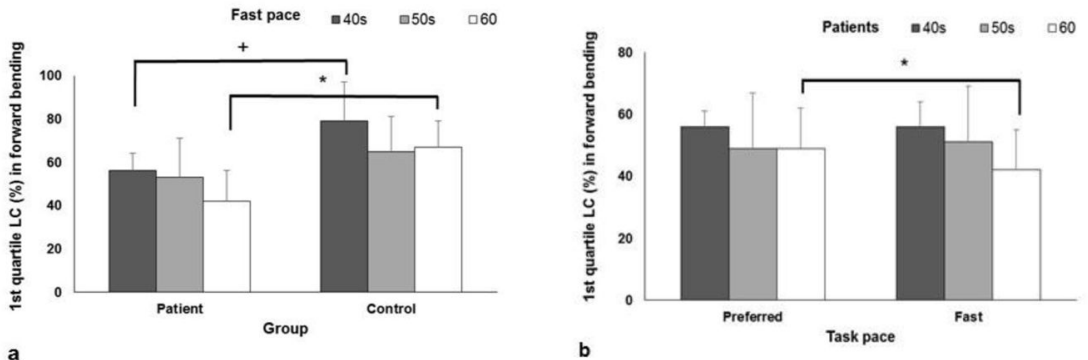

Figure 1.

The simple main effects of group (a) and task pace (b) on lumbar contribution (LC) were significant in the 1st quarter of forward bending. Error bars indicate positive standard deviations. The symbols * and + indicate significant paired differences. 

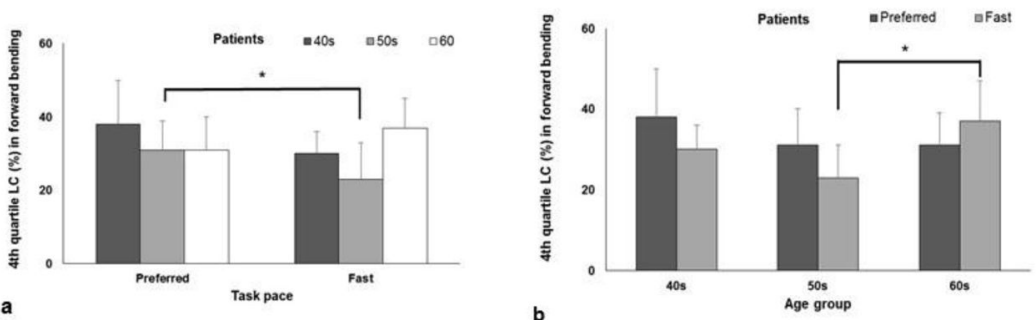

Figure 2.

The simple main effects of task pace (a) and age (b) on lumbar contribution (LC) were significant in the 4th quarter of forward bending. Error bars indicate positive standard deviations. The symbol * indicates significant paired differences. 

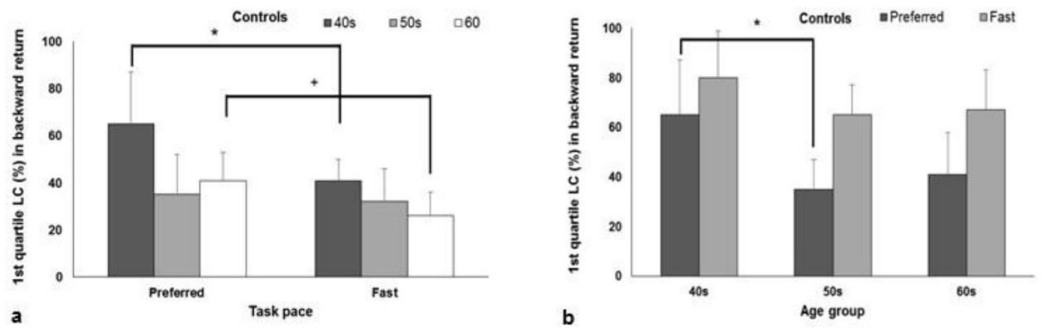

Figure 3.

The simple main effects of task pace (a) and age (b) on lumbar contribution (LC) were significant in the 1st quarter of backward return. Error bars indicate positive standard deviations. The symbols * and + indicate significant paired differences. 

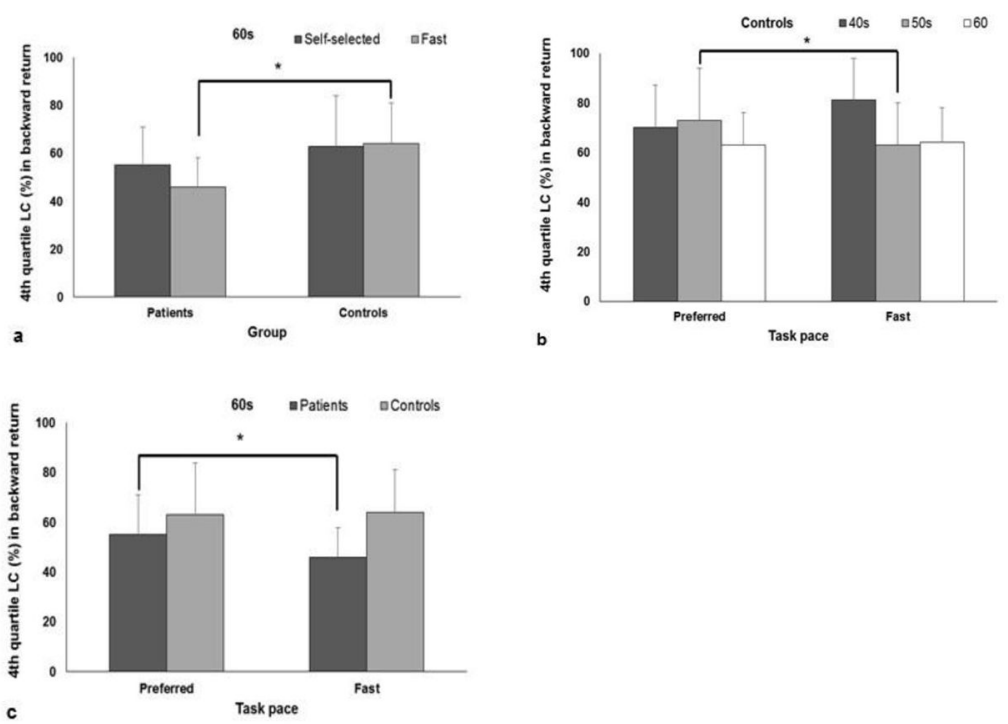

Figure 4.

The simple main effects of group (a) and task pace (b and c) on lumbar contribution (LC) were significant in the 4th quarter of backward return. Error bars indicate positive standard deviations. The symbol * indicates significant paired differences. 


\section{Table 1}

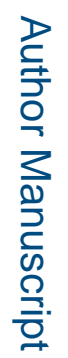

Mean (SD) participants characteristics

\begin{tabular}{ccccc}
\hline Group & Controls & Patients & $\boldsymbol{t}$-value & p-values \\
\hline Age (years) & $56(9)$ & $58(9)$ & 0.723 & 0.474 \\
Stature (m) & $1.64(5)$ & $1.63(7)$ & -0.592 & 0.557 \\
Body mass (kg) & $70(12)$ & $76(17)$ & 1.553 & 0.130 \\
BMI & $25.7(4.1)$ & $27.5(4.6)$ & 1.608 & 0.117 \\
\hline
\end{tabular}




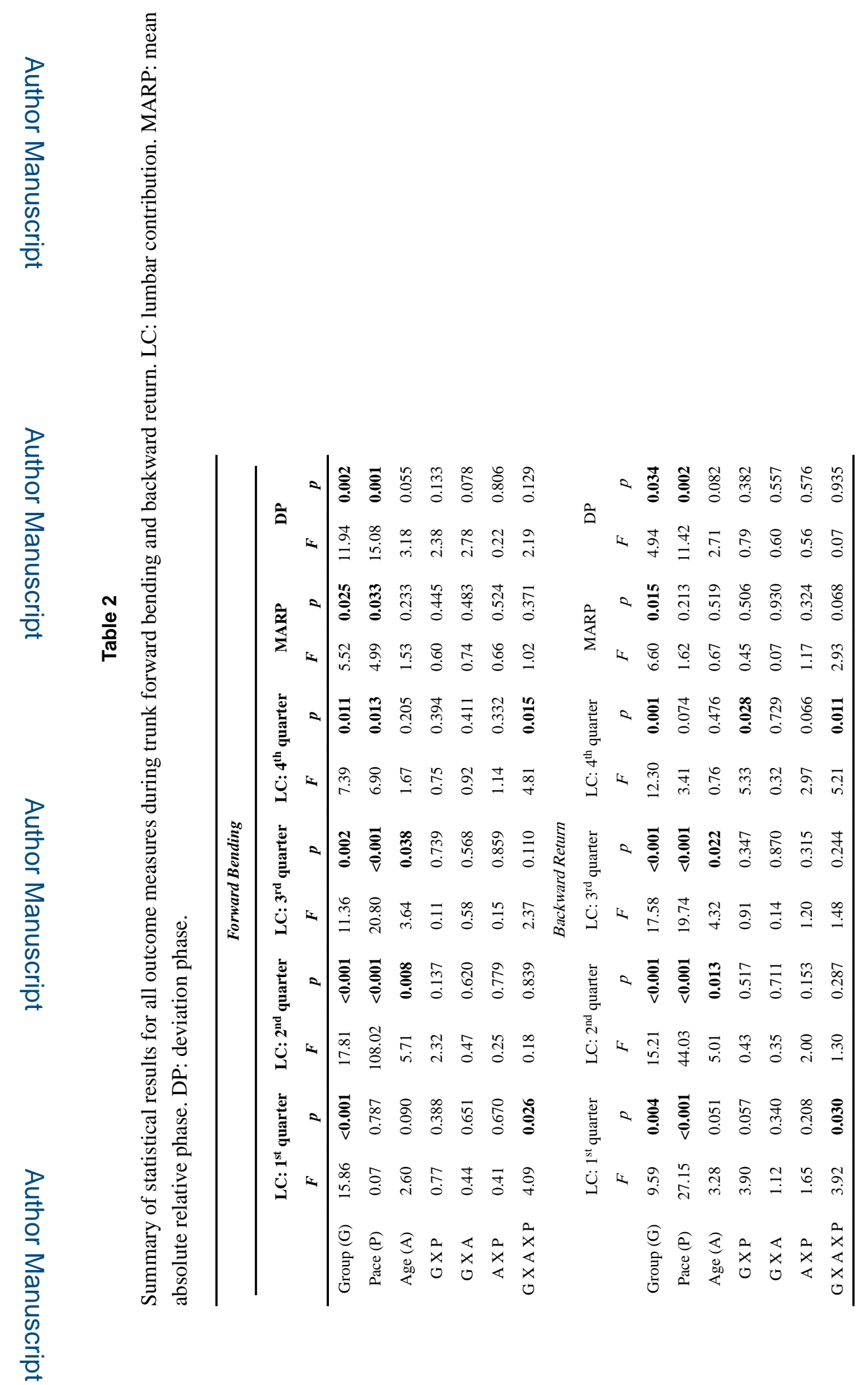

J Biomech. Author manuscript; available in PMC 2018 February 28. 


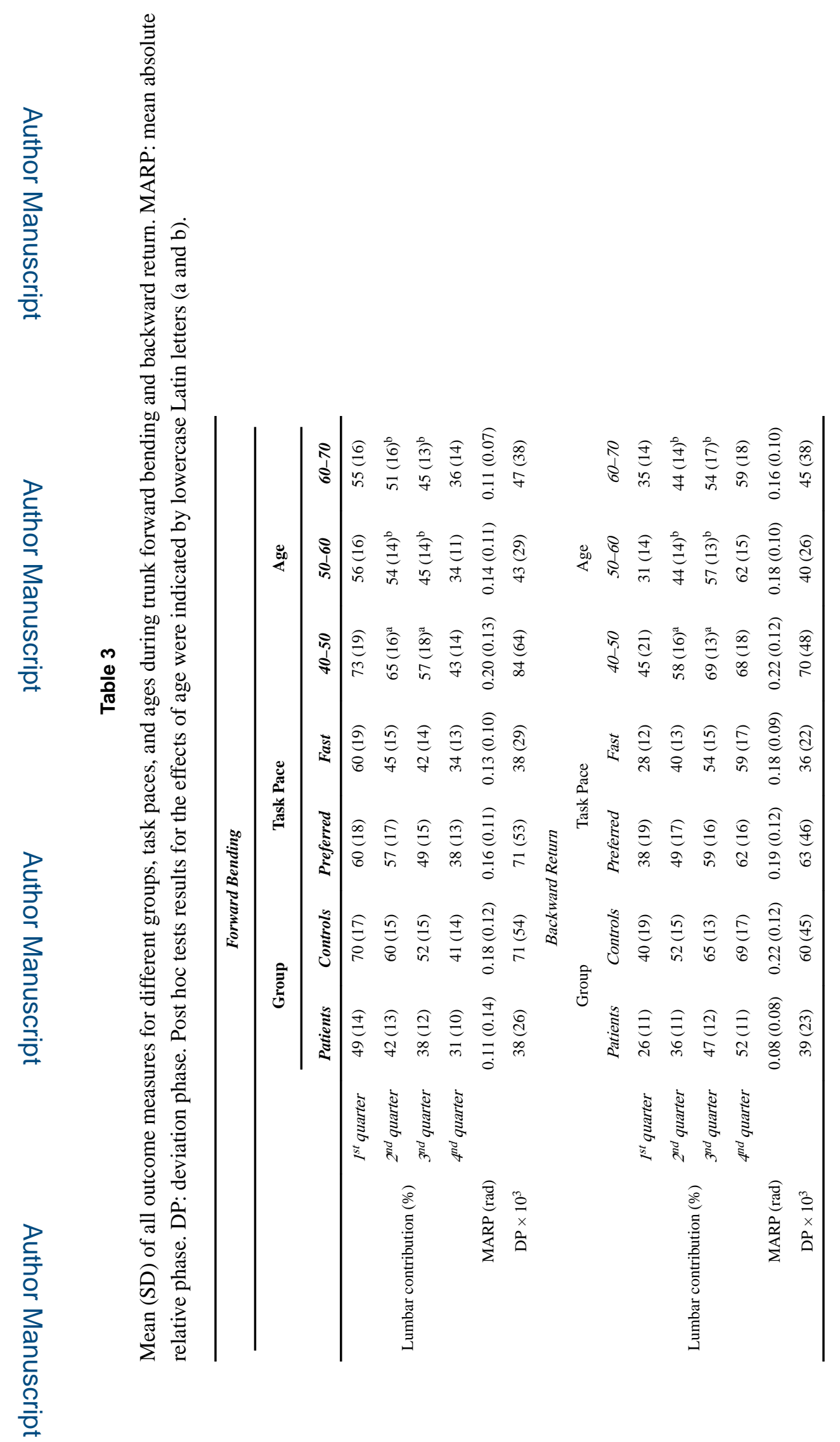

